\title{
Hemispheric contributions to lexical ambiguity resolution in a discourse context: Evidence from individuals with unilateral left and right hemisphere lesions
}

\author{
Christopher M. Grindrod ${ }^{\mathrm{a}, *}$, Shari R. Baum ${ }^{\mathrm{b}}$ \\ a Department of Cognitive and Linguistic Sciences, Brown University, Providence, RI 02912, USA \\ ${ }^{\mathrm{b}}$ School of Communication Sciences and Disorders, Centre for Research on Language, Mind and Brain, McGill University, \\ Montreal, Que., Canada H3G 1 A8
}

Accepted 12 August 2004

\begin{abstract}
In the present study, a cross-modal semantic priming task was used to investigate the ability of left-hemisphere-damaged (LHD) nonfluent aphasic, right-hemisphere-damaged (RHD) and non-brain-damaged (NBD) control subjects to use a discourse context to resolve lexically ambiguous words. Subjects first heard four-sentence discourse passages ending in ambiguous words and after an inter-stimulus interval (ISI) of either 0 or $750 \mathrm{~ms}$, made lexical decisions on first- or second-meaning related visual targets. NBD control subjects, at the $0 \mathrm{~ms}$ ISI, only activated contextually appropriate meanings, though significant effects, as a group, were only seen in second-meaning biased contexts. Surprisingly, at the $750 \mathrm{~ms}$ ISI, these subjects activated both appropriate and inappropriate meanings in first-meaning biased contexts. With respect to the LHD nonfluent aphasic patients, the majority activated first meanings regardless of context at the $0 \mathrm{~ms}$ ISI, though effects for the group were not significant. At the $750 \mathrm{~ms}$ ISI, these patients again activated first meanings regardless of context, with significant effects for the group only seen in first-meaning biased contexts. With regard to the RHD patients, the majority activated second meanings regardless of context at the 0 ms ISI and first meanings regardless of context at the $750 \mathrm{~ms}$ ISI, though, as a group, the effects were not significant. In light of our previous findings (Grindrod \& Baum, 2003, submitted), the present data are interpreted as supporting the notion that damage to the left hemisphere disrupts either lexical access processes or the time course of lexical activation, whereas damage to the right hemisphere impairs the use of context and leads to activation of ambiguous word meanings based on meaning frequency.
\end{abstract}

(C) 2004 Elsevier Inc. All rights reserved.

\section{Introduction}

Though the neural mechanisms underlying language comprehension are assumed to be housed primarily in the left hemisphere $(\mathrm{LH})$, there is now increasing evidence that the right hemisphere ( $\mathrm{RH})$ has an important role to play in language comprehension (for a recent review, see Beeman \& Chiarello, 1998). One particular area in which the two hemispheres have been found to

\footnotetext{
${ }^{*}$ Corresponding author. Fax: +14018632255

E-mail address: christopher_grindrod@brown.edu (C.M. Grindrod).
}

serve complementary functions is in the resolution of lexically ambiguous words (e.g., punch: a beverage and a fist). In large part, lexical ambiguity resolution has served as a window into the functional architecture of the language processing system, to determine whether lexical access is modular (context-independent) or interactive (context-dependent). At present, there is still debate as to whether both meanings of an ambiguous word are initially accessed independent of context (Onifer \& Swinney, 1981; Seidenberg, Tanenhaus, Leiman, \& Bienkowski, 1982; Swinney, 1979; Tanenhaus, Leiman, \& Seidenberg, 1979) or whether only the meaning specific to the context is accessed (Martin, Vu, Kellas, \& 
Metcalf, 1999; Paul, Kellas, Martin, \& Clark, 1992; Simpson \& Krueger, 1991; Tabossi, Colombo, \& Job, 1987; Vu, Kellas, Metcalf, \& Herman, 2000; Vu, Kellas, $\&$ Paul, 1998). While this question continues to underlie current work on ambiguity resolution, much of the attention has now shifted to investigating how the cerebral hemispheres respond to ambiguous words.

Two primary sources of evidence have served as motivation for the hypothesis that the LH and RH make differential contributions to the ambiguity resolution process. Evidence has either come from studies using the divided visual field technique with neurologically intact individuals (Atchley, Burgess, Audet, \& Arambel, 1996; Atchley, Keeney, \& Burgess, 1999; Burgess \& Simpson, 1988; Collins, 2002; Coney \& Evans, 2000; Faust \& Chiarello, 1998; Faust \& Gernsbacher, 1996; Faust \& Kahana, 2002; Faust \& Lavidor, 2003; Hasbrooke \& Chiarello, 1998; Titone, 1998) or from studies of individuals who have suffered focal brain lesions to the LH or RH (Fassbinder \& Tompkins, 2001; Grindrod \& Baum, 2003, submitted; Hagoort, 1990, 1993; Katz, 1988; Milberg, Blumstein, \& Dworetzky, 1987; Prather, Love, Finkel, \& Zurif, 1994; Swaab, Brown, \& Hagoort, 1998; Swinney, Zurif, \& Nicol, 1989; Tompkins, Baumgaertner, Lehman, \& Fassbinder, 2000; Tompkins, Baumgaertner, Lehman, \& Fossett, 1997). ${ }^{1}$ With respect to divided visual field studies, it has been shown that at short stimulus onset asynchronies (SOA), both contextually appropriate and inappropriate meanings of ambiguous words are activated in the two hemispheres (i.e., in the sentence 'He could not wait for even a SECOND,' both the appropriate 'time' and inappropriate 'number' meanings are activated), whereas at longer SOAs, only contextually appropriate meanings are activated in the LH (i.e., only the 'time' meaning), but both appropriate and inappropriate meanings remain activated in the RH (Faust \& Chiarello, 1998; Faust \& Gernsbacher, 1996). From these results, it has been argued that the LH is able to quickly select contextually appropriate meanings of ambiguous words and inhibit inappropriate meanings over time, whereas the $\mathrm{RH}$ serves to maintain alternative meanings, even those that are contextually inappropriate.

With regard to studies of patients with focal brain lesions, it has been shown that individuals with left hemisphere brain damage (LHD), specifically nonfluent Broca's aphasic patients, exhibit a wide range of deficits in resolving ambiguous words in context. The majority of studies have found deficits in these patients' ability to either activate specific meanings of ambiguous words

\footnotetext{
${ }^{1}$ Evidence from another source, fMRI, is just starting to emerge, which may in future allow for the hemispheric mechanisms underlying ambiguity resolution to be more precisely localized (Binzak, Budde, Robertson, Herfel, \& Gernsbacher, 2001; Binzak, Gernsbacher, Budde, \& Kodesh, 2002; Copland et al., 2003).
}

or to select and integrate appropriate meanings into context (Grindrod \& Baum, 2003, submitted; Hagoort, 1990, 1993; Katz, 1988; Milberg et al., 1987; Prather et al., 1994; Swaab et al., 1998; Swinney et al., 1989). With respect to difficulties in activating word meanings, LHD patients, in some cases, have been shown to only activate dominant meanings of ambiguous words, even in contexts biased toward the subordinate meaning (e.g., the 'vegetation' meaning of 'plant' is activated in 'The Ford Motor Company has an assembly PLANT just outside of metropolitan Boston'; Prather et al., 1994; Swinney et al., 1989). This finding implies that either automatic lexical access processes are impaired in these patients or that lexical activation is slowed, such that activation for subordinate meanings is only observed much later in the comprehension process (Prather et al., 1994; Swinney et al., 1989). In other cases, LHD patients have had no difficulty in initially activating ambiguous word meanings, but have been unable to activate meanings at a later point in time, when activation is still observed for neurologically intact subjects (Grindrod \& Baum, 2003; Hagoort, 1993). This finding suggests that activation decays at a faster-than-normal rate in these individuals (Grindrod \& Baum, 2003; Hagoort, 1993). In terms of selection deficits, these patients have been shown to activate both appropriate and inappropriate meanings initially, and then only appropriate meanings at a later-than-normal point in time (Hagoort, 1990; Swaab et al., 1998). This result argues that activation of word meanings is intact in this population and that the problem lies at the level of selecting and integrating meanings into context; most likely, the process of integration is delayed (Hagoort, 1990; Swaab et al., 1998).

Deficits in the ability to resolve ambiguous words in context have also been observed in patients who have suffered right hemisphere brain damage (RHD) (Fassbinder \& Tompkins, 2001; Grindrod \& Baum, 2003, submitted; Tompkins et al., 1997, 2000). In some cases, RHD patients have been shown to experience prolonged interference from inappropriate meanings (e.g., the 'beverage' meaning of 'punch' in 'He landed a PUNCH'; Tompkins et al., 1997, 2000). This finding has been taken as evidence that the mechanism needed to suppress these meanings is impaired in this population (Tompkins et al., 1997, 2000). In contrast to this finding, RHD patients have also been shown to experience no interference from inappropriate meanings initially and considerable interference from these meanings at a later point in time (Fassbinder \& Tompkins, 2001). This result suggests that activation of inappropriate meanings takes much longer to build up and hence, only interferes with ambiguity resolution late in the comprehension process (Fassbinder \& Tompkins, 2001). Finally, RHD patients have also been shown to only activate slightly more frequent meanings, regardless of context (Grindrod \& Baum, 2003, submitted). This finding implies that 
the ability to use context is impaired in these individuals, such that they instead rely on frequency to perform ambiguity resolution (Grindrod \& Baum, 2003, submitted).

In trying to reconcile the findings of brain-damaged patients with those of divided visual field studies, a clear picture has yet to emerge. One hypothesis that remains to be tested is that brain-damaged patients' difficulties in resolving ambiguous words may vary depending on the type of context from which they are required to derive the appropriate meaning. Two recent studies have attempted to address this question by examining ambiguity resolution in both single- and two-sentence contexts (Grindrod \& Baum, 2003, submitted). The motivation behind these studies stems from research showing that LHD patients exhibit improved comprehension in contexts of two or more sentences compared to single-sentence contexts, whereas RHD patients do not demonstrate such marked comprehension improvements in larger contexts (Cannito, Jarecki, \& Pierce, 1986; Hough, 1990; Hough, Pierce, \& Cannito, 1989; Wapner, Hamby, \& Gardner, 1981). It is, therefore, of empirical interest to examine whether a single-sentence versus a multiple-sentence context differentiates among LHD and RHD patients in terms of their ability to resolve ambiguity. In one study, Grindrod and Baum (2003) presented groups of LHD and RHD individuals and non-brain-damaged (NBD) control subjects with ambiguous words in biased single-sentence (local) contexts (e.g., After writing a long message, he looked at the CARD [greeting vs. playing card]). Subjects performed a cross-modal lexical decision task, designed to measure the extent to which ambiguous word meanings were primed, at both short $(0 \mathrm{~ms})$ and long $(750 \mathrm{~ms})$ inter-stimulus intervals (ISI). Of particular note was the finding that both patient groups had difficulty using the local contextual information to resolve the ambiguous words. LHD nonfluent aphasic patients primed both meanings regardless of context at the short ISI and no meanings at the long ISI. In contrast, RHD patients, for the most part, only primed slightly more frequent meanings at both ISIs. Thus, neither patient group appeared to be sensitive to a single-sentence biasing context.

To assess these patients' sensitivity to a somewhat larger context, Grindrod and Baum (submitted) conducted another study in which ambiguous words were embedded in a two-sentence (global) context (e.g., The driver examined the rust. After he spotted a hole, he repaired the HOOD [car vs. jacket hood]). The results of this experiment confirmed those of Grindrod and Baum (2003), showing that brain-damaged patients were no more sensitive to larger contextual information. In fact, the performance of the LHD nonfluent aphasic patients was much worse, in that they were unable to prime any meanings at either a short or long ISI. Similar to the pre- vious study, RHD patients again only showed priming for more frequent meanings at both ISIs. Thus, deficits comparable in nature to those seen in a single-sentence context were observed in both LHD and RHD patients, a finding which runs counter to the prediction that LHD patients might be better able to resolve ambiguity in larger contexts, whereas RHD patients may not be so wellequipped in that regard. Interestingly, findings for the LHD patients from these two studies are in large part consistent with previous proposals of impaired activation of ambiguous word meanings or faster-than-normal decay of activation (Hagoort, 1993; Prather et al., 1994; Swinney et al., 1989). Findings for the RHD patients, however, fail to support previous proposals of a deficient suppression mechanism or slowed activation of inappropriate meanings (Fassbinder \& Tompkins, 2001; Tompkins et al., 1997, 2000).

In an attempt to examine whether an even larger context (in this case, a four-sentence discourse passage) would better differentiate among the LHD and RHD patient groups in terms of their ability to perform ambiguity resolution, the present study was undertaken. A discourse context may be the most sensitive measure of the ability to use context to resolve ambiguity, as the biasing information is not in close proximity to the ambiguous word and must therefore be integrated across a number of sentences. Moreover, having this distance between the biasing information and the ambiguous word may allow more time for integration to take place or for activation of a particular meaning to build up from the context. In the current study, a cross-modal semantic priming task was employed, as in our previous investigations (Grindrod \& Baum, 2003, submitted). LHD nonfluent aphasic, RHD, and NBD control subjects first listened to biased four-sentence discourse passages ending in lexically ambiguous (or unambiguous control) words and then made lexical decisions on visually presented targets, related to either the first or second meaning of the ambiguity. In each passage, the first sentence (i.e., discourse context) was always biased toward one meaning of the ambiguity and the last sentence (i.e., local context) was unbiased. The two intermediary sentences were neutral with respect to either meaning of the ambiguous words and provided a congruent link between the biasing and ambiguity-bearing sentences. To examine the time course of activation of ambiguous word meanings, both a short $(0 \mathrm{~ms})$ and long $(750 \mathrm{~ms})$ ISI were employed.

NBD control subjects, if they are sensitive to constraints provided by the discourse context, should only show priming for contextually appropriate meanings at both the short and long ISIs (Martin et al., 1999; Vu et al., 1998, 2000). LHD nonfluent aphasic patients, if they are able to automatically access ambiguous word meanings, but exhibit a delay in integrating these meanings into context, are expected to show priming for both 
meanings regardless of context at the short ISI and only for contextually appropriate meanings at the long ISI (Swaab et al., 1998). Alternatively, if these patients are unable to access word meanings, they should fail to show priming at either ISI (Swinney et al., 1989). Moreover, if they exhibit a faster-than-normal decay of activation, they should demonstrate priming for both meanings of the ambiguous words at the short ISI and no priming at the long ISI (Grindrod \& Baum, 2003; Hagoort, 1993). RHD patients, if they are unable to use context and instead rely on frequency to perform ambiguity resolution, are expected to only activate slightly more frequent meanings regardless of context at both the short and long ISIs (Grindrod \& Baum, 2003, submitted). Alternatively, if they are unable to suppress inappropriate meanings, they should show priming for both meanings regardless of context at both ISIs (Tompkins et al., 1997, 2000). Finally, if these patients experience slower-than-normal activation of inappropriate meanings, they should show more priming for appropriate than inappropriate meanings at the short ISI and comparable amounts of priming for the two meanings at the long ISI (Fassbinder \& Tompkins, 2001).

\section{Method}

\subsection{Subjects}

Three groups of subjects participated in this study: 10 LHD nonfluent aphasic individuals, eight RHD individuals, and nine age- and education-matched non-braindamaged (NBD) control subjects. ${ }^{2}$ Participants were all right-handed, native speakers of English with normal or corrected-to-normal vision. Hearing for all subjects was found to be within normal limits as determined by a hearing screening at $<35 \mathrm{~dB} \mathrm{HL}$ in the better ear at the speech frequencies $0.5,1.0$, and $2.0 \mathrm{kHz}$.

Exclusionary criteria for the brain-damaged patients included: (1) the presence of multiple infarcts, (2) a known history of drug or alcohol abuse, and (3) a history of psychiatric and/or other neurological illness. All patients had suffered a single left or right cerebrovascular accident (CVA) and were at least 6 months postonset at the time of testing. Lesion sites were determined based on neurological reports and radiological summaries of Computerized Cranial Tomography (CT) and/ or Magnetic Resonance Imaging (MRI) scans where

\footnotetext{
${ }^{2}$ Initially, 12 LHD nonfluent aphasic patients and 12 NBD control subjects were tested. The data from two patients were excluded, as these individuals had extremely high error rates. The data from three control subjects were also excluded, either due to these subjects having mean reaction times more than two standard deviations slower than the overall group mean (two subjects) or to testing error (one subject).
}

available. Patients were diagnosed based on their clinical evaluations and their performance on a variety of screening tests. These tests included: (1) the Behavioural Inattention Test (BIT) (Wilson, Cockburn, \& Halligan, 1987) to assess visual neglect, (2) the auditory word-picture matching (AWPM) subtest of the Psycholinguistic Assessment of Language (PAL) (Caplan, 1992) to assess single word reading comprehension, (3) the auditory sentence comprehension (ASC) subtest of the PAL (Caplan, 1992) to assess comprehension of spoken sentences varying in syntactic complexity, (4) the auditory working memory (AWM) task (Tompkins, Bloise, Timko, \& Baumgaertner, 1994) to assess memory for single words ${ }^{3}$ and (5) the Discourse Comprehension Test (DCT) (Brookshire \& Nicholas, 1993) to assess comprehension of spoken discourse. In addition, LHD nonfluent aphasic individuals were administered a number of subtests of the Boston Diagnostic Aphasia Examination (BDAE) (Goodglass \& Kaplan, 1983; Goodglass, Kaplan, \& Barresi, 2001) to assess their language abilities. RHD individuals were administered tests adapted from the Test of Language Competence-Expanded Edition (TLC-E) (Wiig \& Secord, 1989) to assess their ability to generate inferences and to understand figurative language, skills that are frequently impaired in this population. The patient groups did not differ in the number of months post-onset (MPO) of stroke $[F(1,16)=0.537$, $p=.474]$, nor did they differ in terms of their performance on the BIT $[F(1,14)=.266, p=.614]$. Not surprisingly, they did perform differently on the auditory sentence comprehension (ASC) subtest of the PAL. Specifically, while the two groups performed equally well on semantically constrained sentences $[F(1,16)=2.433$, $p=.138]$, the LHD nonfluent aphasic individuals performed much worse than the RHD individuals on semantically reversible sentences $[F(1,16)=8.739$, $p<.01]$. Background information for the LHD and RHD individuals is presented in Table 1.

NBD control subjects had no history of drug or alcohol abuse or of neurological or psychiatric illness. To rule out the possibility of cognitive decline or dementia, they were screened on a series of neuropsychological tests that included: (1) the Boston Naming Test (BNT) (Kaplan, Goodglass, \& Weintraub, 1983), (2) the Mini-Mental State Examination (MMSE) (Folstein, Folstein, \& McHugh, 1975), and (3) the Logical Memory I

\footnotetext{
${ }^{3}$ As in Tompkins et al. (1994), because of the demands the auditory working memory task places on spoken word recall, it was not administered to LHD nonfluent aphasic subjects who produced fewer than five concepts from the Yorkston and Beukelman (1980) list, when describing the "Cookie Theft" picture from the BDAE (Goodglass \& Kaplan, 1983). Of the three patients who produced fewer than five concepts, two were also unable to complete the BNT due to their expressive limitations. In addition, two of the three patients had relatively low percentiles on the auditory comprehension subtest of the BDAE.
} 
Table 1

Brain-damaged patients' background information

\begin{tabular}{|c|c|c|c|c|c|c|c|c|c|c|c|c|c|c|c|}
\hline \multicolumn{16}{|c|}{ Left-hemisphere-damaged nonfluent aphasic patients } \\
\hline \multirow[t]{3}{*}{$\overline{\text { Patient }}$} & \multirow{3}{*}{$\begin{array}{l}\text { Age } \\
\text { (years) }\end{array}$} & \multirow[t]{3}{*}{ Sex } & \multirow{3}{*}{$\begin{array}{l}\text { Education }{ }^{\mathrm{a}} \\
\text { (years) }\end{array}$} & \multirow[t]{3}{*}{ Etiology } & \multirow[t]{3}{*}{ Lesion site $^{\mathrm{b}}$} & \multirow[t]{3}{*}{ MPO } & \multirow{3}{*}{$\begin{array}{l}\text { BDAE } \\
\text { AC } \\
(\text { mean \%ile) }\end{array}$} & \multirow[t]{3}{*}{ BNT (60) } & \multicolumn{3}{|l|}{ PAL } & \multirow[t]{3}{*}{ DCT (40) } & \multicolumn{2}{|c|}{ AWM (errors) } & \multirow[t]{3}{*}{$\operatorname{BIT}^{\mathrm{c}}(146)$} \\
\hline & & & & & & & & & \multirow{2}{*}{$\overline{\text { SWPM }}$} & \multicolumn{2}{|l|}{ ASC } & & \multirow{2}{*}{ Recall (42) } & \multirow{2}{*}{$\mathrm{T} / \mathrm{F}(42)$} & \\
\hline & & & & & & & & & & $\overline{\mathrm{C}}$ & $\mathrm{R}$ & & & & \\
\hline 1 & 57 & $\mathrm{M}$ & 10 & I & $\mathrm{L}$ fronto-temporo-parietal & 33 & 84 & 39 & 1.00 & 0.95 & 0.90 & 31 & 29 & 2 & $\mathrm{n} / \mathrm{a}$ \\
\hline 2 & 54 & M & 14 & I & L parietal & 176 & 90 & 51 & 1.00 & 0.95 & 0.65 & 38 & 20 & 4 & 146 \\
\hline 3 & 70 & $\mathrm{~F}$ & 11 & I & L fronto-parietal & 86 & 83 & 18 & 1.00 & 0.85 & 0.65 & 36 & 30 & 2 & 145 \\
\hline 4 & 71 & $\mathrm{~F}$ & 9 & $\mathrm{H}$ & L fronto-temporo-parietal & 112 & 60 & 11 & 1.00 & 0.85 & 0.50 & 32 & $\mathrm{n} / \mathrm{a}$ & $\mathrm{n} / \mathrm{a}$ & 143 \\
\hline 5 & 81 & $\mathrm{~F}$ & 16 & I & L parietal & 28 & 60 & 26 & 0.94 & 0.85 & 0.60 & 30 & 37 & 4 & 132 \\
\hline 6 & 50 & $\mathrm{~F}$ & 15 & I & $\mathrm{L}$ fronto-parietal & 130 & 88 & 44 & 0.97 & 0.95 & 0.85 & 35 & 22 & 6 & 146 \\
\hline 7 & 82 & $\mathrm{M}$ & 9 & I & $\mathrm{L}$ frontal & 89 & 89 & 55 & 0.94 & 1.00 & 0.70 & 36 & 16 & 2 & 145 \\
\hline 8 & 74 & $\mathrm{~F}$ & 12 & I & $\mathrm{L}$ parietal & 113 & 89 & 52 & 1.00 & 1.00 & 1.00 & 37 & 20 & 0 & 141 \\
\hline 9 & 61 & M & 20 & $\mathrm{n} / \mathrm{a}$ & CT scan negative $<1$ day & 45 & 30 & $\mathrm{n} / \mathrm{a}$ & 0.97 & 0.85 & 0.70 & 34 & $\mathrm{n} / \mathrm{a}$ & $\mathrm{n} / \mathrm{a}$ & $\mathrm{n} / \mathrm{a}$ \\
\hline 10 & 76 & $\mathrm{M}$ & 12 & I & $\mathrm{L}$ temporo-parietal & 148 & 93 & $\mathrm{n} / \mathrm{a}$ & 0.97 & 1.00 & 0.75 & $\mathrm{n} / \mathrm{a}$ & $\mathrm{n} / \mathrm{a}$ & $\mathrm{n} / \mathrm{a}$ & 143 \\
\hline$M$ & 67.60 & & 12.80 & & & 96.00 & 76.60 & 37.00 & 0.98 & 0.93 & 0.73 & 34.33 & 24.86 & 2.86 & 142.63 \\
\hline$S D$ & 11.38 & & 3.49 & & & 49.61 & 20.29 & 16.72 & 0.03 & 0.07 & 0.15 & 2.78 & 7.36 & 1.95 & 4.63 \\
\hline \multicolumn{16}{|c|}{ Right-hemisphere-damaged patients } \\
\hline \multirow[t]{2}{*}{ Patient } & \multirow{2}{*}{$\begin{array}{l}\text { Age } \\
\text { (years) }\end{array}$} & \multirow[t]{2}{*}{ Sex } & \multirow{2}{*}{$\begin{array}{l}\text { Education }{ }^{\mathrm{a}} \\
\text { (years) }\end{array}$} & \multirow[t]{2}{*}{ Etiology } & \multirow[t]{2}{*}{ Lesion site $^{\mathrm{b}}$} & \multirow[t]{2}{*}{ MPO } & \multicolumn{2}{|l|}{ TLC-E } & PAL AS & & DCT (40) & AWM (erro & & $\operatorname{BIT}^{\mathrm{c}}(146)$ & \\
\hline & & & & & & & Figurative & $\overline{\text { Inferences }}$ & $\overline{\mathrm{C}}$ & $\mathrm{R}$ & & Recall (42) & $\mathrm{T} / \mathrm{F}(42)$ & & \\
\hline 1 & 72 & M & 14 & $\mathrm{H}$ & $\mathrm{R}$ thalamus (subcortical) & 72 & 0.60 & 0.60 & 0.95 & 0.90 & 36 & 19 & 0 & 144 & \\
\hline 2 & 65 & M & 12 & I & $\mathrm{R}$ corona radiata (subcortical) & 25 & 0.70 & 0.60 & 0.90 & 0.80 & 37 & 1 & 0 & 136 & \\
\hline 3 & 36 & $\mathrm{~F}$ & 13 & I & $\mathrm{R}$ parietal & 92 & 0.90 & 1.00 & 1.00 & 0.90 & 35 & 14 & 1 & 146 & \\
\hline 4 & 90 & M & 11 & $\mathrm{H}$ & $\mathrm{n} / \mathrm{a}$ & 73 & 0.70 & 0.60 & 1.00 & 0.90 & 35 & 22 & 2 & 141 & \\
\hline 5 & 61 & $\mathrm{~F}$ & 13 & $\mathrm{H}$ & R PCA distribution & 151 & 0.80 & 1.00 & 1.00 & 0.95 & 36 & 13 & 1 & 146 & \\
\hline 6 & 68 & $\mathrm{~F}$ & 13 & $\mathrm{H}$ & R basal ganglia (subcortical) & 92 & 0.50 & 0.90 & 1.00 & 0.95 & 36 & 12 & 1 & 143 & \\
\hline 7 & 81 & M & 11 & $\mathrm{H}$ & $\mathrm{R}$ fronto-temporo-parietal & 67 & 0.70 & 0.70 & 1.00 & 0.85 & 33 & 16 & 1 & 129 & \\
\hline 8 & 45 & $\mathrm{~F}$ & 9 & I & R MCA distribution & 74 & 0.60 & 0.40 & 0.90 & 0.90 & 34 & 13 & 0 & 145 & \\
\hline$M$ & 64.75 & & 12.00 & & & 80.75 & 0.69 & 0.73 & 0.97 & 0.89 & 35.25 & 13.75 & 0.75 & 141.25 & \\
\hline$S D$ & 17.71 & & 1.60 & & & 35.19 & 0.12 & 0.22 & 0.05 & 0.05 & 1.28 & 6.18 & 0.71 & 5.95 & \\
\hline
\end{tabular}

Note. AC, Auditory Comprehension; ASC, Auditory Sentence Comprehension; AWM, Auditory Working Memory (Tompkins et al., 1994); BDAE, Boston Diagnostic Aphasia Examination (Goodglass and Kaplan, 1983; Goodglass et al., 2001); BIT, Behavioural Inattention Test (Wilson et al., 1987); BNT, Boston Naming Test (Kaplan et al., 1983); C, Constrained; DCT, Discourse Comprehension Test (Brookshire and Nicholas, 1993); H, Hemorrhage; I, Infarct; MCA, Middle cerebral artery; MPO, Months post-onset; n/a, Information not available; PAL, Psycholinguistic Assessment of Language (Caplan, 1992); PCA, Posterior cerebral artery; R, Reversible; SWPM, Single Word-Picture Matching; TLC-E, Test of Language Competence-Expanded Edition (Wiig and Secord, 1989).

${ }^{a}$ Best estimated conversion into years, based on information from subject (e.g., 2 years college, high school).

b Established based on CT/MRI scan and/or neurological reports.

c Neglect cutoff $=129$. 
Table 2

Example of experimental stimuli

\begin{tabular}{|c|c|c|c|}
\hline \multirow[t]{2}{*}{ Biasing context } & \multirow[t]{2}{*}{ Discourse context prime } & \multicolumn{2}{|l|}{ Visual target } \\
\hline & & 1st meaning & 2nd meaning \\
\hline 1st meaning biased & $\begin{array}{l}\text { The athlete headed for the locker room. He was really tired from the long night. } \\
\text { He walked slowly up the steps. When he opened the door, he saw the BAT (THIEF). }\end{array}$ & Ball & Cave \\
\hline 2nd meaning biased & $\begin{array}{l}\text { The man heard a noise in the attic. He was really tired from the long night. } \\
\text { He walked slowly up the steps. When he opened the door, he saw the BAT (THIEF). }\end{array}$ & Ball & Cave \\
\hline
\end{tabular}

Note. Control words given in parentheses replaced ambiguous words in control passages.

(immediate recall) and II (delayed recall) subtests of the Wechsler Memory Scale-Revised (WMS-R) (Wechsler, 1987). The age of the control subjects $(M=71.33$, $S D=3.91)$ was no different from that of the brain-damaged subjects $[F(2,24)=0.641, p=.536]$. This was also the case with respect to the number of years of education of the control $(M=13.67, S D=2.40)$ and brain-damaged subjects $[F(2,24)=0.816, p=.454]$. A significant difference between the groups was found on the DCT $[F(2,23)=4.28, p<.05]$, with post hoc tests using the Tukey method indicating that this effect was due to the lower scores of the LHD nonfluent aphasic patients compared to the NBD subjects. There was also a significant difference in performance on the AWM task, in terms of the number of word recall errors $[F(2,21)=17.217, \quad p<.0001]$ and true/false errors $[F(2,21)=11.067, p<.001]$. Post hoc tests using the Tukey method revealed that the LHD nonfluent aphasic subjects made significantly more word recall and true/ false errors than the RHD and NBD subjects.

\subsection{Materials}

Forty-one ambiguous words, each having two meanings of approximately equal frequency (i.e., equibiased or balanced), were initially selected from Twilley, Dixon, Taylor, and Clark (1994). ${ }^{4}$ Equibiased ambiguous words were chosen in an attempt to decrease the likelihood of frequency-driven meaning selection, so that the effects of context on ambiguity resolution could be for the most part isolated. The ambiguous words were selected according to the following criteria: (1) they were all noun-noun ambiguities, (2) the frequency of occurrence of the first meaning was never greater than .70, and (3) the frequency of occurrence of the second meaning was at least .20 (in order to exclude very infrequent meanings).

For each ambiguous word, two biasing passages were created, adapted from those used in Grindrod and Baum (submitted). The stimuli were short four-sentence

\footnotetext{
${ }^{4}$ In most cases, even equibiased ambiguous words have one meaning which is slightly more frequent. In the present study, the first meaning of an equibiased ambiguous word is equated with the somewhat more frequent (i.e., dominant) meaning and the second meaning with the somewhat less frequent (i.e., subordinate) meaning.
}

discourse passages, in which the biasing information was always contained in the first sentence (i.e., the discourse context) and the ambiguous word in the final unbiased sentence (i.e., the local context). To create a short discourse, two filler sentences were inserted between the biasing sentence and the ambiguity-bearing sentence (see example stimuli provided in Table 2). These filler sentences were congruent continuations of the initial sentence and were neutral with respect to the two meanings of the ambiguous words. Control conditions were constructed by replacing passage-final ambiguous words with unambiguous control words while keeping all other elements of the passages identical (see Table 2). These unambiguous control words were selected to form congruent completions to the final sentence in each passage. They were also matched to the ambiguous words for frequency (Francis \& Kucera, 1982) and length. The ambiguous words had a mean frequency of 101.10 and the unambiguous control words, a mean frequency of $137.80[t(19)=0.849, p=.406]$.

Two pretests were conducted on the materials. One on the final sentence of the four-sentence discourse passage to ensure that it was unbiased and another on the biasing and ambiguity-bearing sentences together to ensure that the first sentence created the appropriate bias (for further details regarding these pretests, refer to Grindrod \& Baum, submitted). Results of the pretests led to the selection of 20 ambiguous words that met the following criteria: (1) for the unbiased final sentence, not more than $70 \%$ of subjects' responses were related to one of the two meanings, thus ensuring that one meaning was not heavily favoured, (2) for the biasing passage, at least $70 \%$ of subjects' responses were related to the intended meaning biased by the context, ensuring that the context was indeed biased in the right direction, and (3) for control passages, not more than $30 \%$ of subjects' responses were related to either meaning, ensuring that the context itself did not make one meaning more predictable than the other. For the ambiguous words selected as experimental items, the first meaning had a mean frequency of .50 (range: .30-.70), while the second meaning had a mean frequency of .30 (range: .20-.42). These values are comparable to those of equibiased ambiguous words used in other studies (Binder \& Morris, 1995; Duffy, Morris, \& Rayner, 1988; Rayner \& Duffy, 1986; Rayner \& Frazier, 1989; Sereno, 1995). In terms of the 
bias of the passages, $90 \%$ (range: $71-100 \%$ ) of subjects' responses were related to the intended meaning for first-meaning biased contexts, whereas $89 \%$ (range: $73-$ $100 \%$ ) of subjects' responses were related to the intended meaning for second-meaning biased contexts.

Two associates for each ambiguous word were selected from the Twilley et al. (1994) or Nelson, McEvoy, and Schreiber (1998) norms to serve as visual targets. Each target was paired with both ambiguity- and control-bearing passages (see Table 2). To avoid the development of strategies by subjects, an equal number of filler passages were also constructed, half of which ended in an ambiguous word and the other half in an unambiguous word. These passages were similar in length and style to the critical passages. Following the presentation of filler passages, a pronounceable nonword was presented for lexical decision. Nonwords were approximately matched to word targets for length and were orthographically legal letter strings. All passages were recorded by a female speaker of English, digitized at a rate of $20 \mathrm{~K}$ samples/s and low pass filtered at $9 \mathrm{kHz}$ using the Brown Lab Interactive Speech System (BLISS) software (Mertus, 2000).

Eight lists (four per ISI) were created such that neither auditory priming passages nor visual targets were repeated within a list. Each list contained 20 ambiguity-bearing passages with word targets, 20 control-bearing passages with word targets, and 40 filler passages with nonword targets ( 80 trials in total). Trials within each list were presented in a pseudorandom order, with the restriction that there was a maximum of three word or nonword lexical decisions in succession.

\subsection{Procedure}

All subjects were tested in four sessions of approximately $1 \mathrm{~h}$ each (two sessions per ISI). Within each session, subjects were presented with two lists, in a blocked design. Order of presentation of the lists and ISI were counterbalanced across all subjects. Within each ISI condition, the two sessions were separated by at least 1 week (to minimize effects of repetition). In addition, at least 1 month separated the two ISI conditions.

Each trial began with the presentation of an auditory four-sentence discourse passage through headphones. At 0 or $750 \mathrm{~ms}$ after the offset of the passage-final ambiguous or control word, a visual target was displayed in the center of the computer screen. Subjects were instructed to make a lexical decision response on the visual target by pressing the YES button of the mouse for a word and the NO button for a nonword (using their currently dominant hand). The computer recorded both reaction time (in ms) and accuracy. Reaction time was recorded from the onset of the visual target until the subject responded. After $4000 \mathrm{~ms}$ without a response, the trial was recorded as a no-response.
The inter-trial interval was $5000 \mathrm{~ms}$. A practice list of 10 trials preceded presentation of the experimental session lists. In cases where subjects did not understand the task, the practice list was repeated. To ensure that subjects listened to the passages, they were asked to answer yes/no comprehension questions on a random $10 \%$ of the trials (i.e., eight comprehension questions per list). Comprehension questions were only asked about filler passages.

\section{Results}

Accuracy rates for lexical decision responses were first examined to identify and exclude data from stimulus lists with unusually high error rates. For each subject, accuracy rates were calculated for each of the four experimental lists in each ISI condition. Examining accuracy rates within a particular list seemed justified, as subjects may have had a high accuracy rate overall, but not necessarily within all lists. Moreover, as each list was administered separately, error rates could have potentially varied among the lists (especially within the patient groups). A cutoff rate of $67 \%$ was used, such that any list where a subject made more than $33 \%$ errors was removed. This criterion resulted in the removal of one list for an RHD individual (S4) in the 750 ms ISI condition. In terms of the distribution of errors, all subject groups made less than 3\% real word errors and less than $4.5 \%$ nonword errors, showing that they were able to perform the task with a high degree of accuracy. NBD control subjects made a total of $0.10 \%$ real word errors (3/ 2880 trials) and $0.49 \%$ nonword errors (14/2880 trials). LHD nonfluent aphasic individuals made a total of $0.91 \%$ real word errors (29/3200 trials) and $4.44 \%$ nonword errors (142/3200 trials), while RHD individuals made a total of $2.62 \%$ real word errors $(66 / 2520$ trials) and $3.73 \%$ nonword errors $\left(94 / 2520\right.$ trials). ${ }^{5}$ Given that the distribution of real word errors (control versus related) did not differentiate among the subject groups and that accuracy did not vary as a function of any of the experimental conditions in this study, only the results of the reaction time (RT) data will be discussed.

Statistical analyses were conducted on the RTs for correct responses to real word targets. RTs greater than $2500 \mathrm{~ms}$ or 2.5 SDs above each subject's mean per condition and RTs less than $250 \mathrm{~ms}$ or 2.5 SDs below each subject's mean per condition were treated as outliers and excluded from further analyses. This resulted in the elimination of $2.64 \%$ of the data for NBD control subjects, $2.78 \%$ for LHD nonfluent aphasic subjects, and $3.18 \%$ for RHD individuals. Before conducting the data analysis, a z-score transformation was applied to each

\footnotetext{
${ }^{5}$ In the RHD group, a number of real word errors were produced by one subject (S4).
} 
$0 \mathrm{~ms}$ ISI

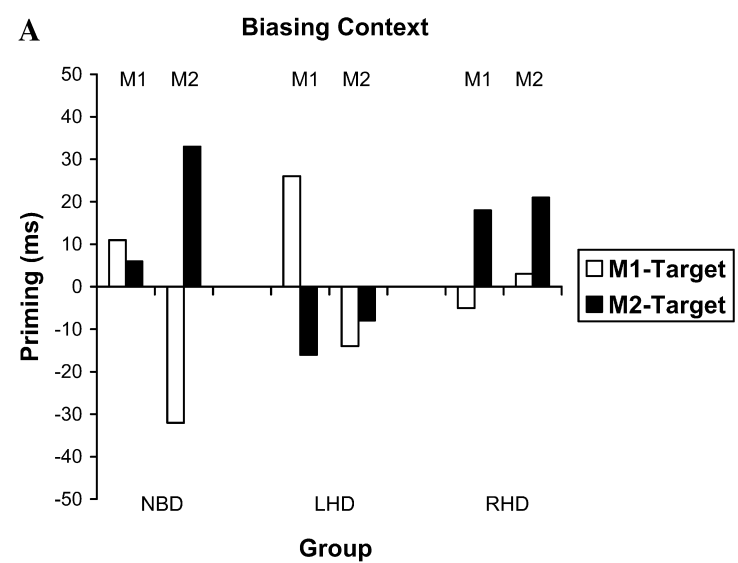

$750 \mathrm{~ms}$ ISI

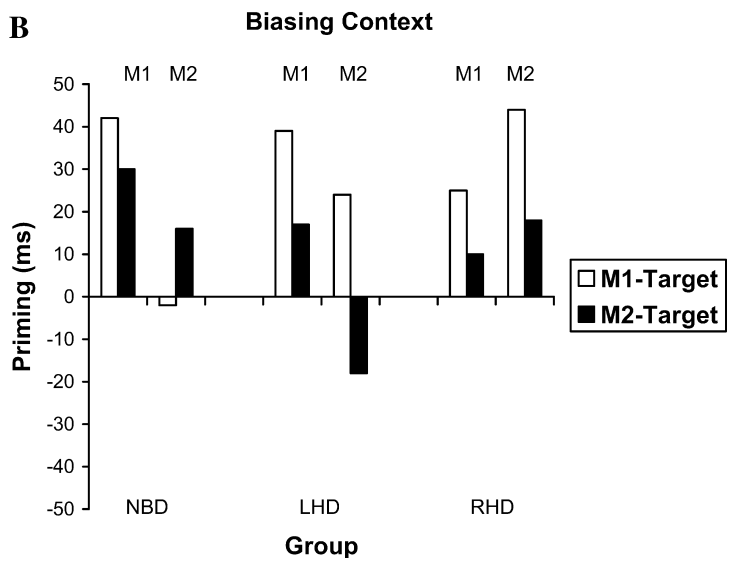

Fig. 1. Priming $\left[\mathrm{RT}_{\text {control }}-\mathrm{RT}_{\text {related }}\right]$ at the 0 and $750 \mathrm{~ms}$ ISI. M1, first meaning; M2, second meaning. NBD, non-brain-damaged control subjects; LHD, left-hemisphere-damaged patients; RHD, right-hemisphere-damaged patients.

subjects' mean RT in each condition to correct for differences in processing speed (see Faust, Balota, Spieler, \& Ferraro, 1999). A $2 \times 2 \times 2$ (Biasing Context $\times$ Prime Relatedness $\times$ Target Type) multivariate repeated measures analysis of variance (MANOVA) with subjects (F1) and items (F2) as random factors was then conducted on the z-transformed RT data for each group and ISI condition. In addition, planned pairwise comparisons of related and control conditions were conducted on each group's data in order to determine whether or not priming was observed for targets related to the first or second meanings of the ambiguous words in each condition (see Hagoort, 1990; Swinney et al., 1989; for the rationale behind this type of analysis). Priming effects for each group per condition and ISI are presented in Fig. 1.

\section{1. $0 \mathrm{~ms} I S I$}

For the NBD control subjects at the $0 \mathrm{~ms}$ ISI, there was a significant main effect of Target Type
$[F 1(1,8)=9.751, p<.05 ; F 2(1,19)=3.082, p=.095]$ and a significant interaction of Prime Type $\times$ Target Type, though only in the subject analyses $[F 1(1,8)=5.296, p<.05 ; F 2(1,19)=3.651, p=.071]$. There was also a significant interaction of Biasing Context $\times$ Prime Type $\times$ Target Type in the item analysis $[F 1(1,8)=3.418, p=.102 ; F 2(1,19)=5.773, p<.05]$. No other main effects or interactions were found to be significant. Planned comparisons between related and control conditions revealed that priming was only found for targets related to the contextually appropriate meaning in second-meaning biased contexts $[F(1,8)=7.446$, $p<.05]$. Inspection of the individual subject data revealed that $67 \%$ of subjects showed priming for the appropriate meaning in this context. In first-meaning biased contexts, no significant priming effects for the group were observed. Despite the lack of a significant effect, $67 \%$ of the individual subjects did show priming for the contextually appropriate first meaning in this context.

For the LHD nonfluent aphasic patients, there was a significant main effect of Biasing Context in the subject analysis $[F 1(1,9)=10.349, p<.05 ; F 2(1,19)=4.070$, $p=.058]$. No other main effects or interactions were found to be significant. Planned comparisons failed to reveal any significant priming effects for the group in either biasing context, though a number of patients did show priming. In terms of the individual subject data, in first-meaning biased contexts, $70 \%$ of the patients showed priming for the contextually appropriate first meaning, a comparable number to the NBD control subjects. Interestingly, in second-meaning biased contexts, $50 \%$ of the patients also showed priming for the first meaning, though inappropriate in this context.

For the RHD patients, no main effects or interactions were found to be significant. In addition, planned comparisons failed to reveal any significant priming effects for the group in either context. Though effects for the group were not significant, an interesting pattern of results did emerge when the individual subject data were inspected. Specifically, the majority of patients appeared to show priming for the second meaning regardless of context. In first-meaning biased contexts, $63 \%$ of the patients showed priming for the contextually inappropriate second meaning, whereas $88 \%$ of the patients showed priming for the appropriate second meaning in secondmeaning biased contexts. The fact that these effects did not reach significance could have been due to either the large variability in the individual patients' RTs (range: $600-1500 \mathrm{~ms}$ ), to the small number of patients in this group or to the impact of two patients' large effects in the unexpected direction (i.e., control RT faster than the related RT).

In sum, NBD control subjects appeared to be influenced by the discourse context at the $0 \mathrm{~ms}$ ISI. Though significant priming effects, as a group, were only found 
for appropriate meanings in second-meaning biased contexts, the majority of subjects also showed priming for the contextually appropriate meaning in first-meaning biased contexts. With respect to the patients, no significant priming effects were obtained in the group data; however, the individual subject data did reveal an interesting pattern of results. A large number of LHD nonfluent aphasic patients showed priming for first meanings regardless of context. In contrast, the majority of RHD patients showed priming for second meanings regardless of context.

\section{2. $750 \mathrm{~ms} I S I$}

For NBD control subjects at the $750 \mathrm{~ms}$ ISI, there were significant main effects of Biasing Context $[F 1(1,8)=12.638, p<.01 ; F 2(1,19)=20.755, p<.001]$ and Prime Type $[F 1(1,8)=10.248, \quad p<.05$; $F 2(1,19)=6.123, p<.05]$. No other main effects or interactions were found to be significant. Planned comparisons revealed that in first-meaning biased contexts, both targets related to the contextually appropriate first meaning $[F(1,8)=50.538, p<.001]$ and to the inappropriate second meaning $[F(1,8)=9.361, p<.05]$ were primed. With respect to the individual subject data, $89 \%$ of subjects showed priming for the appropriate meaning in this context and $78 \%$ for the inappropriate meaning. In second-meaning biased contexts, no significant priming effects for the group were observed. Despite the lack of a significant effect, $44 \%$ of the subjects showed priming for the appropriate second meaning, a much smaller number than at the $0 \mathrm{~ms}$ ISI.

For the LHD nonfluent aphasic patients, there was a significant main effect of Prime Type in the item analysis $[F 1(1,9)=3.255, p=.105 ; F 2(1,19)=5.114, p<.05]$. No other main effects or interactions were found to be significant. Planned comparisons revealed that in firstmeaning biased contexts, only targets related to the appropriate first meaning were primed $[F(1,9)=7.199$, $p<.05]$. In terms of the individual subject data, this effect was observed in $70 \%$ of the patients. In secondmeaning biased contexts, no significant priming effects for the group were produced, though $70 \%$ of the individual patients did show priming for targets related to the contextually inappropriate first meaning, a number comparable in nature to that seen in the $0 \mathrm{~ms}$ ISI condition.

For the RHD patients, there was a significant main effect of Prime Type in the subject analysis $[F 1(1,7)=7.535, p<.05 ; F 2(1,19)=2.876, p=.106]$. No other main effects or interactions were found to be significant. Planned comparisons also revealed no significant priming effects for the group, for targets related to first or second meanings in either context. With respect to the individual subject data, the majority of RHD patients appeared to be priming first meanings regardless of context. Specifically, in both first- and second-meaning biased contexts, $63 \%$ of the patients showed priming for targets related to the first meaning. Again, the fact that these results did not reach conventional levels of significance may have been due to the small number of patients or to the large variability in the individual patients' RTs (range: $550-1650 \mathrm{~ms}$ ).

In sum, results for the NBD control subjects at the $750 \mathrm{~ms}$ ISI were somewhat unexpected. While these subjects showed priming for the contextually appropriate meaning in first-meaning biased contexts, they also demonstrated priming for the inappropriate second meaning. Also surprising was the finding that less than half of the subjects showed priming for the appropriate meaning in second-meaning biased contexts. With respect to the LHD nonfluent aphasic patients, they again only primed first meanings, a finding consistent with the individual subject data at the $0 \mathrm{~ms}$ ISI. Finally, the RHD patients, as a group, produced no significant priming effects, similar to what was seen at the $0 \mathrm{~ms}$ ISI. Interestingly, while the majority of RHD patients showed priming for second meanings regardless of context at the 0 ms ISI, most of the patients showed priming for first meanings regardless of context at the $750 \mathrm{~ms}$ ISI.

\section{Discussion}

This study set out to investigate the extent to which patients with LH or RH lesions and non-brain-damaged control subjects were able to use a biased four-sentence discourse context to resolve ambiguous words. NBD control subjects appeared to be sensitive to the context at the $0 \mathrm{~ms}$ ISI, though, as a group, selective activation of the contextually appropriate meaning was only seen in second-meaning biased contexts. Crucially, the majority of subjects also only activated the appropriate meaning in first-meaning biased contexts. At the $750 \mathrm{~ms}$ ISI, control subjects, as a group, unexpectedly activated both appropriate and inappropriate meanings in firstmeaning biased contexts. In second-meaning biased contexts, a number of individuals showed priming for the appropriate meaning, though, as a group, the effect was not significant. An account of the surprising result at the $750 \mathrm{~ms}$ ISI is presented below. LHD nonfluent aphasic patients, as a group, produced no significant activation of ambiguous word meanings at the $0 \mathrm{~ms}$ ISI, though a number of patients activated first meanings regardless of context. At the $750 \mathrm{~ms}$ ISI, these patients again activated first meanings regardless of context, though the effect for the group was only significant in first-meaning biased contexts. This pattern of results suggests that the patients had difficulty accessing second meanings, which may indicate that either lexical access processes are disrupted or the time course of lex- 
ical activation is delayed (Milberg et al., 1987; Prather et al., 1994; Swinney et al., 1989). RHD individuals, as a group, produced no significant activation at either ISI, though an interesting pattern of results did emerge when looking at the individual patient data. Specifically, the majority of patients activated second meanings regardless of context at the $0 \mathrm{~ms}$ ISI and first meanings regardless of context at the $750 \mathrm{~ms}$ ISI. While results at the $0 \mathrm{~ms}$ ISI were surprising, findings at the $750 \mathrm{~ms}$ ISI fit well with previous data showing that RHD patients perform ambiguity resolution based on frequency (Grindrod \& Baum, 2003, submitted).

At both the 0 and $750 \mathrm{~ms}$ ISI, NBD control subjects were expected to only activate discourse-appropriate meanings, assuming they were sensitive to constraints imposed by the context (Martin et al., 1999; Simpson, 1994; Vu et al., 1998, 2000). In line with this prediction, control subjects in the present study demonstrated selective activation of contextually appropriate meanings, as a group, in second-meaning biased contexts at the $0 \mathrm{~ms}$ ISI. In addition, the majority of individual subjects only activated contextually appropriate meanings in firstmeaning biased contexts, with the effect for the group in the right direction, but failing to reach significance. Somewhat surprising was the finding that at the $750 \mathrm{~ms}$ ISI, the control subjects, as a group, activated appropriate as well as inappropriate meanings in firstmeaning biased contexts. In second-meaning biased contexts, a number of subjects only activated appropriate meanings, though the effect for the group was again not significant.

The most surprising finding for the elderly control subjects was that activation for both appropriate and inappropriate meanings was observed in first-meaning biased contexts at the $750 \mathrm{~ms}$ ISI. This was unexpected, given that previous studies of normal young subjects have shown that appropriate meanings are selected relatively quickly (and inappropriate meanings presumably have decayed or have been suppressed), usually within $200 \mathrm{~ms}$ after the ambiguous word is encountered (for a review, see Simpson, 1994). Under certain conditions, inappropriate meanings, even those that are less frequent, have been found to be activated at later points in time, if, for example, the context is unbiased (Simpson \& Krueger, 1991) or if it is not highly constraining or weakly biased (Binder \& Rayner, 1998; Martin et al., 1999; Rayner, Patchi, \& Duffy, 1994). Admittedly, evidence for later activation of inappropriate meanings in younger subjects is rather limited, given that many of these studies, using eye-tracking and self-paced reading methodologies, do not provide a direct indication as to how long the inappropriate meaning remains activated. Still, it is clear from these subjects' longer reading times in the region immediately following the ambiguous word, that both appropriate and inappropriate meanings can be activated for some time after the ambiguous word is encountered (Binder \& Rayner, 1998; Rayner et al., 1994).

Though the results at the $750 \mathrm{~ms}$ ISI do not fit well with previous findings for young subjects, it is important to note that at least one recent study, similar in design to the present one, has found activation for both meanings in older subjects at a longer ISI (Titone, Leonard, \& Baum, 2004). In Titone et al. (2004), older subjects were found to activate more frequent dominant meanings in less-frequent subordinate-biased contexts at an ISI of $1250 \mathrm{~ms}$. These results, along with the present data, could be interpreted as arguing for the possibility of an age-related decline in the ability to inhibit or suppress inappropriate meanings (Hartman \& Hasher, 1991; Hasher \& Zacks, 1988). At present, more data are needed to confirm this proposal, especially given that other studies have shown no difference between older and younger subjects in the ability to inhibit contextually inappropriate meanings (Hopkins, Kellas, \& Paul, 1995; Paul, 1996).

As an alternative proposal, rather than attributing the present results to an age-related difference, it could be that specific aspects of the context, as alluded to above, played a role. In the present study, the contexts were not only less-constrained, but they also contained an unbiased sentence, both of which could have led to activation of inappropriate meanings at the $750 \mathrm{~ms}$ ISI. With respect to the contexts being less-constrained, recall that the biasing information was far-removed from the occurrence of the ambiguous word, therefore it is possible that this information did not exert such a strong influence on processing. Moreover, as the local (final-sentence) context was unbiased, this information could have also overridden the biasing information from the discourse context. Though a possible explanation, this proposal does go against recent research by Kambe, Rayner, and Duffy (2001), who showed that young subjects are influenced by the discourse context and attempt to use this information, despite the presence of potentially conflicting local contextual information. Thus, the proposal that the distance of the biasing discourse context and the unbiased nature of the local context interacted to produce activation for both meanings in the current study awaits further empirical validation.

With respect to the LHD nonfluent aphasic patients, they produced no significant activation, as a group, at the $0 \mathrm{~ms}$ ISI, though the majority of individual patients activated first meanings regardless of context. At the $750 \mathrm{~ms}$ ISI, the patients, as a group, activated appropriate meanings in first-meaning biased contexts and no meanings in second-meaning biased contexts, though again the majority of patients also activated inappropriate first meanings in this context. As such, the present findings are in keeping with the prediction that these individuals have difficulty accessing ambiguous word meanings or are slow to activate second meanings (Milberg et al., 1987; Prather et al., 1994; Swinney et al., 
1989). Moreover, though the patients did not appear to be consistently and effectively using the discourse context, the present data further suggest that it did have some effect on this group's ability to activate word meanings. In particular, the larger context seems to have helped boost activation of first meanings, such that a consistent number of the patients activated these meanings at the $750 \mathrm{~ms}$ ISI, a greater number of patients than seen in our two previous studies using shorter contexts (Grindrod \& Baum, 2003, submitted).

In terms of the nonfluent aphasic patients' data, the most interesting finding was that these individuals activated first meanings at the $750 \mathrm{~ms}$ ISI. This result was unexpected given that in our recent investigations, where the context was varied from either a single sentence to two sentences (Grindrod \& Baum, 2003, submitted), we did not find any activation at a $750 \mathrm{~ms}$ ISI with a similar group of patients. When we consider other findings, however, it is less surprising that activation, as a group, was not observed until late in the comprehension process. It has been well-documented that nonfluent aphasic patients are slow to activate word meanings, taking as long as $1500 \mathrm{~ms}$ to activate meanings in some studies (Prather et al., 1994; Prather, Zurif, Love, \& Brownell, 1997; Prather, Zurif, Stern, \& Rosen, 1992; Swinney et al., 1989). It is particularly noteworthy that patients in the current study were able to activate meanings at $750 \mathrm{~ms}$, a point much earlier in time than noted in the studies by Prather and colleagues. What may have allowed the patients to activate meanings somewhat earlier is the additional processing time afforded by the lengthier discourse context. Not only did the patients have an additional $750 \mathrm{~ms}$, but they also had more time for lexical-semantic activation to build up, as the discourse context unfolded. As no benefits of additional processing time, in the form of a longer ISI, were noted in our two previous studies with shorter contexts (Grindrod \& Baum, 2003, submitted), it seems plausible that the combination of the longer discourse context and the longer ISI provided the nonfluent aphasic patients with even more processing time, which ultimately contributed to their ability to activate first meanings. As an aside, results at the $0 \mathrm{~ms}$ ISI also point to the fact that the discourse context was exerting an influence on the patients' ability to activate word meanings. Even at this early point, a number of patients activated first meanings, though, as a group, the effects were not significant. Thus, it seems that based on the discourse context alone, the patients were able to activate first meanings, though only at a subthreshold (or weak) level. With the additional processing time afforded by the $750 \mathrm{~ms}$ ISI and the longer discourse context, first meanings appeared to receive a boost in activation, such that they reached threshold by $750 \mathrm{~ms}$.

Turning finally to the RHD patients, these individuals, as a group, failed to activate ambiguous word mean- ings at either ISI, a finding inconsistent with the predictions made at the outset of the present investigation. Despite the lack of significant effects for the group, the individual subject data did reveal a striking pattern of results. At the $0 \mathrm{~ms}$ ISI, the majority of individuals activated second meanings regardless of context. In contrast, at the $750 \mathrm{~ms}$ ISI, the majority of individuals activated first meanings regardless of context. While findings at the $0 \mathrm{~ms}$ ISI are inconsistent with our previous research (Grindrod \& Baum, 2003, submitted), findings at the $750 \mathrm{~ms}$ ISI are in line with our previous work, in which RHD patients have been shown to rely on meaning frequency to perform ambiguity resolution (Grindrod \& Baum, 2003, submitted).

Most surprising about the RHD patients' results was that an unexpectedly large number of individuals activated second meanings at the $0 \mathrm{~ms}$ ISI. Of our recent investigations (Grindrod \& Baum, 2003, submitted), this is the first time activation for second meanings has been observed in this group (except for a nonsignificant trend seen in Grindrod \& Baum, 2003). As such, these data are difficult to reconcile with any previous accounts. It cannot be the case that the patients were using frequency to resolve the ambiguous words, nor can it be that they were using the context. At present, there is no obvious explanation for this unexpected pattern of results and we can only conclude that they are aberrant in nature. It is, however, important to note that the priming observed for second meanings was primarily due to one patient, who produced rather large priming effects for this meaning at the $0 \mathrm{~ms}$ ISI. With respect to this individual, there are no apparent characteristics which set him apart from the rest of the group, taking into consideration lesion location in the $\mathrm{RH}$, months post-onset of stroke, scores on the language tests administered or other demographic variables. Still, this individual was highly influential in producing the surprising pattern of results at the $0 \mathrm{~ms}$ ISI.

In contrast to the unexpected findings at the $0 \mathrm{~ms}$ ISI, results at the $750 \mathrm{~ms}$ ISI were quite consistent with our previous data (Grindrod \& Baum, 2003, submitted). In this condition, the majority of individuals activated first meanings regardless of context, with results for the group just failing to reach significance. Disregarding the findings at the $0 \mathrm{~ms}$ ISI, these data lend support to the hypothesis that RHD patients use frequency to resolve ambiguity, rather than the context (Grindrod \& Baum, 2003, submitted). Moreover, they further argue against recent claims by Tompkins and colleagues that RHD individuals cannot suppress inappropriate meanings (Tompkins et al., 1997, 2000) or activate these meanings at a slower-than-normal rate (Fassbinder \& Tompkins, 2001). With regard to these alternative proposals, it is particularly noteworthy that the patients in the current study always activated one meaning more than the other. The key assumption underlying the work of Tompkins and colleagues is that for inappropriate 
meanings to interfere with comprehension, appropriate meanings must also be activated; however, activation of appropriate meanings is never explicitly demonstrated in their studies, as only targets related to the inappropriate meaning are presented. In the current experiment, a greater proportion of RHD individuals always activated one meaning over the other, thus inappropriate meanings never seemed to be activated along with appropriate meanings, casting some doubt on the claim that these individuals have difficulty suppressing inappropriate meanings.

\section{Conclusion}

Given the variability of the present results and the aberrant findings, caution must be taken in drawing conclusions from the current study. Nevertheless, the results, for the most part, do confirm those of previous investigations indicating that individuals with $\mathrm{LH}$ or $\mathrm{RH}$ lesions exhibit deficits in the ability to resolve ambiguous words in context. In light of our previous findings (Grindrod \& Baum, 2003, submitted), the present data further argue that LH damage disrupts lexical access mechanisms and/or the time course of lexical-semantic activation. They are also consistent, in large part, with the proposal that RH damage impairs the ability to use biasing contextual information, leading ambiguity resolution to be performed based on meaning frequency. Interestingly, the discourse context, though originally intended to differentiate among the two patient groups in terms of their ability to perform ambiguity resolution, failed to set one group of patients apart from the other in the present study. It did, however, appear to exert some influence on the patients' ability to activate word meanings. Though significant activation, as a group, was only found for the LHD nonfluent aphasic patients, a large number of individuals in both patient groups activated one or the other meaning of the ambiguous words. This is particularly telling, given that a much smaller number of individual patients were able to activate ambiguous word meanings in our previous studies using shorter contexts (Grindrod \& Baum, 2003 , submitted). In sum, the present study provides preliminary evidence that a larger context may contribute to brain-damaged patients' ability to activate word meanings, though it does not necessarily allow them to fully exploit the biasing information. Future research should continue to assess the impact of other contextual manipulations on brain-damaged patients' ability to perform the complex operation of lexical ambiguity resolution.

\section{Acknowledgments}

The research reported here forms part of a doctoral dissertation by C.M. Grindrod submitted to McGill Univer- sity. This work was supported by a Doctoral Research Award from the Canadian Institutes of Health Research to C.M. Grindrod and by a research grant from the Canadian Institutes of Health Research to S.R. Baum. The authors would like to thank Meg Grant, Pablo Ruiz, and Lisa Trayhern for their assistance in collecting the brain-damaged patients' data and Wendi Aasland for recording the stimuli. We are also indebted to the patients for their continued interest and participation and to the speech-language pathologists at the local hospitals and rehabilitation centers for their efforts in identifying and recruiting participants.

\section{References}

Atchley, R. A., Burgess, C., Audet, C., \& Arambel, S. (1996). Timecourse, context effects and the processing of lexical ambiguity in the cerebral hemispheres. Brain and Cognition, 30, 277-280.

Atchley, R. A., Keeney, M., \& Burgess, C. (1999). Cerebral hemispheric mechanisms linking ambiguous word meaning retrieval and creativity. Brain and Cognition, 40, 479-499.

Beeman, M. J., \& Chiarello, C. (Eds.). (1998). Right hemisphere language comprehension: Perspectives from cognitive neuroscience. Mahwah, NJ: Lawrence Erlbaum Associates.

Binder, K. S., \& Morris, R. K. (1995). Eye movements and lexical ambiguity resolution: Effects of prior encounter and discourse topic. Journal of Experimental Psychology: Learning, Memory and Cognition, 21, 1186-1196.

Binder, K. S., \& Rayner, K. (1998). Contextual strength does not modulate the subordinate bias effect: Evidence from eye fixations and self-paced reading. Psychonomic Bulletin and Review, 5, 271-276.

Binzak, J. B., Budde, M. D., Robertson, D. A., Herfel, H. G., \& Gernsbacher, M. A. (2001, March). Suppression of irrelevant meanings of homographs. Poster presented at the annual meeting of the Cognitive Neuroscience Society, New York, NY.

Binzak, J. B., Gernsbacher, M. A., Budde, M. D., \& Kodesh, G. T. (2002, June). The role of the right hemisphere in successful comprehension of homonyms. Poster presented at the annual meeting of the Society for Text and Discourse, Chicago, IL.

Brookshire, R. H., \& Nicholas, L. E. (1993). Discourse comprehension test. Tucson, AZ: Communication Skill Builders.

Burgess, C., \& Simpson, G. B. (1988). Cerebral hemispheric mechanisms in the retrieval of ambiguous word meanings. Brain and Language, 33, 86-103

Cannito, M. P., Jarecki, J. M., \& Pierce, R. S. (1986). Effects of thematic structure on syntactic comprehension in aphasia. Brain and Language, 27, 38-49.

Caplan, D. (1992). Language: Structure, process and disorders. Cambridge: MIT Press.

Collins, M. (2002). Interhemispheric communication via direct connections for alternative meanings of ambiguous words. Brain and Language, 80, 77-96.

Coney, J., \& Evans, K. D. (2000). Hemispheric asymmetries in the resolution of lexical ambiguity. Neuropsychologia, 38, 272-282.

Copland, D. A., de Zubicaray, G. I., McMahon, K., Wilson, S. J., Eastburn, M., \& Chenery, H. J. (2003). Brain activity during automatic semantic priming revealed by event-related functional magnetic resonance imaging. Neuroimage, 20, 302-310.

Duffy, S. A., Morris, R. K., \& Rayner, K. (1988). Lexical ambiguity and fixation times in reading. Journal of Memory and Language, 27, 429-446. 
Fassbinder, W., \& Tompkins, C. A. (2001). Slowed lexical-semantic activation in individuals with right hemisphere brain damage? Aphasiology, 15, 1079-1090.

Faust, M., \& Chiarello, C. (1998). Sentence context and lexical ambiguity resolution by the two hemispheres. Neuropsychologia, $36,827-835$.

Faust, M., \& Kahana, A. (2002). Priming summation in the cerebral hemispheres: Evidence from semantically convergent and semantically divergent primes. Neuropsychologia, 40, 892-901.

Faust, M., \& Lavidor, M. (2003). Semantically convergent and semantically divergent priming in the cerebral hemispheres: Lexical decision and semantic judgment. Cognitive Brain Research, 17, $585-597$.

Faust, M. E., Balota, D. A., Spieler, D. H., \& Ferraro, F. R. (1999). Individual differences in information-processing rate and amount: Implications for group differences in response latency. Psychological Bulletin, 125, 777-799.

Faust, M. E., \& Gernsbacher, M. A. (1996). Cerebral mechanisms for suppression of inappropriate information during sentence comprehension. Brain and Language, 53, 234-259.

Folstein, M. F., Folstein, S. E., \& McHugh, P. R. (1975). "Mini-mental state": A practical method for grading the cognitive state of patients for the clinician. Journal of Psychiatric Research, 12, 189-198.

Francis, W., \& Kucera, H. (1982). Frequency analysis of English usage. Boston, MA: Houghton Mifflin.

Goodglass, H., \& Kaplan, E. (1983). Boston diagnostic aphasia examination (2nd ed.). Philadelphia: Lea and Febiger.

Goodglass, H., Kaplan, E., \& Barresi, B. (2001). Boston diagnostic aphasia examination (3rd ed.). Philadelphia: Lippincott Williams and Wilkins.

Grindrod, C. M., \& Baum, S. R. (2003). Sensitivity to local sentence context information in lexical ambiguity resolution: Evidence from left- and right-hemisphere-damaged individuals. Brain and Language, 85, 503-523.

Grindrod, C. M., \& Baum, S. R. (submitted). Global context effects on lexical ambiguity resolution in left- and right-hemisphere-damaged individuals. Journal of Neurolinguistics.

Hagoort, P. (1990). Tracking the time course of language understanding in aphasia. Ph.D. dissertation, University of Nijmegen, The Netherlands.

Hagoort, P. (1993). Impairments of lexical-semantic processing in aphasia: Evidence from the processing of lexical ambiguities. Brain and Language, 45, 189-232.

Hartman, M., \& Hasher, L. (1991). Aging and suppression: Memory for previously relevant information. Psychology and Aging, 6, 587-594.

Hasbrooke, R. E., \& Chiarello, C. (1998). Bihemispheric processing of redundant bilateral lexical information. Neuropsychology, 12, 78-94.

Hasher, L., \& Zacks, R. T. (1988). Working memory, comprehension, and aging: A review and a new view. In G. H. Bower (Ed.). The psychology of learning and motivation: Advances in research and theory (Vol. 22, pp. 193-225). San Diego: Academic Press.

Hopkins, K. A., Kellas, G., \& Paul, S. T. (1995). Scope of word meaning activation during sentence processing by young and older adults. Experimental Aging Research, 21, 123-142.

Hough, M. S. (1990). Narrative comprehension in adults with right and left hemisphere brain-damage: Theme organization. Brain and Language, 38, 253-277.

Hough, M. S., Pierce, R. S., \& Cannito, M. P. (1989). Contextual influences in aphasia: Effects of predictive versus nonpredictive narratives. Brain and Language, 36, 325-334.

Kambe, G., Rayner, K., \& Duffy, S. A. (2001). Global context effects on processing lexically ambiguous words: Evidence from eye fixations. Memory and Cognition, 29, 363-372.

Kaplan, E., Goodglass, H., \& Weintraub, S. (1983). Boston naming test. Philadelphia: Lea and Febiger.
Katz, W. F. (1988). An investigation of lexical ambiguity in Broca's aphasics using an auditory lexical priming technique. Neuropsychologia, 26, 747-752.

Martin, C., Vu, H., Kellas, G., \& Metcalf, K. (1999). Strength of discourse context as a determinant of the subordinate bias effect. Quarterly Journal of Experimental Psychology A, 52, 813-839.

Mertus, J. (2000). Brown lab interactive speech system [computer software]. Providence, RI: Brown University.

Milberg, W., Blumstein, S. E., \& Dworetzky, B. (1987). Processing of lexical ambiguities in aphasia. Brain and Language, 31, $138-150$.

Nelson, D. L., McEvoy, C. L., Schreiber, T. A. (1998). The University of South Florida word association, rhyme, and word fragment norms. Available from : w3.usf.edu/FreeAssociation/.

Onifer, W., \& Swinney, D. A. (1981). Accessing lexical ambiguities during sentence comprehension: Effects of frequency of meaning and contextual bias. Memory and Cognition, 9, 225-236.

Paul, S. T. (1996). Search for semantic inhibition failure during sentence comprehension by younger and older adults. Psychology and Aging, 11, 10-20.

Paul, S. T., Kellas, G., Martin, M., \& Clark, M. B. (1992). Influence of contextual features on the activation of ambiguous word meanings. Journal of Experimental Psychology: Learning, Memory and Cognition, 18, 703-717.

Prather, P. A., Love, T., Finkel, L., \& Zurif, E. (1994). Effects of slowed processing on lexical activation: Automaticity without encapsulation. Brain and Language, 47, 326-329.

Prather, P. A., Zurif, E., Love, T., \& Brownell, H. H. (1997). Speed of lexical activation in nonfluent Broca's aphasia and fluent Wernicke's aphasia. Brain and Language, 59, 391-411.

Prather, P. A., Zurif, E., Stern, C., \& Rosen, T. J. (1992). Slowed lexical access in nonfluent aphasia: A case study. Brain and Language, 43, 336-348.

Rayner, K., \& Duffy, S. A. (1986). Lexical complexity and fixation times in reading: Effects of word frequency, verb complexity, and lexical ambiguity. Memory and Cognition, 14, 191-201.

Rayner, K., \& Frazier, L. (1989). Selection mechanisms in reading lexically ambiguous words. Journal of Experimental Psychology: Learning, Memory and Cognition, 15, 779-790.

Rayner, K., Pacht, J. M., \& Duffy, S. A. (1994). Effects of prior encounter and global discourse bias on the processing of lexically ambiguous words: Evidence from eye fixations. Journal of Memory and Language, 33, 527-544.

Seidenberg, M. S., Tanenhaus, M. K., Leiman, J. M., \& Bienkowski, M. (1982). Automatic access of the meanings of ambiguous words in context: Some limitations of knowledge-based processing. Cognitive Psychology, 14, 489-537.

Sereno, S. C. (1995). Resolution of lexical ambiguity: Evidence from an eye movement priming paradigm. Journal of Experimental Psychology: Learning, Memory and Cognition, 21, 582-595.

Simpson, G. B. (1994). Context and the processing of ambiguous words. In M. A. Gernsbacher (Ed.), Handbook of psycholinguistics (pp. 359-374). New York: Academic Press.

Simpson, G. B., \& Krueger, M. A. (1991). Selective access of homograph meanings in sentence context. Journal of Memory and Language, 30, 627-643.

Swaab, T. Y., Brown, C. M., \& Hagoort, P. (1998). Understanding ambiguous words in sentence contexts: Electrophysiological evidence for delayed contextual selection in Broca's aphasia. Neuropsychologia, 36, 737-761.

Swinney, D. A. (1979). Lexical access during sentence comprehension: (Re)consideration of context effects. Journal of Verbal Learning and Verbal Behaviour, 18, 645-659.

Swinney, D. A., Zurif, E., \& Nicol, J. L. (1989). The effects of focal brain damage on sentence processing: An examination of the neurological organization of a mental module. Journal of Cognitive Neuroscience, 1, 25-37. 
Tabossi, P., Colombo, L., \& Job, R. (1987). Accessing lexical ambiguity: Effects of context and dominance. Psychological Research, 49, 161-167.

Tanenhaus, M. K., Leiman, J. M., \& Seidenberg, M. S. (1979). Evidence for multiple stages in the processing of ambiguous words in syntactic contexts. Journal of Verbal Learning and Verbal Behaviour, 18, $427-440$.

Titone, D. A. (1998). Hemispheric differences in context sensitivity during lexical ambiguity resolution. Brain and Language, 65, 361-394.

Titone, D. A., Leonard, C. L., \& Baum, S. R. (2004, July). Lexical ambiguity resolution in older adults: When a failure to inhibit is adaptive? Paper presented at the Fourth International Conference on the Mental Lexicon, Windsor, Ontario, Canada.

Tompkins, C. A., Baumgaertner, A., Lehman, M. T., \& Fassbinder, W. (2000). Mechanisms of discourse comprehension impairment after right hemisphere brain damage: Suppression in lexical ambiguity resolution. Journal of Speech, Language and Hearing Research, 43, 62-78.

Tompkins, C. A., Baumgaertner, A., Lehman, M. T., \& Fossett, T. R. D. (1997). Suppression and discourse comprehension in right braindamaged adults: A preliminary report. Aphasiology, 11, 505-519.

Tompkins, C. A., Bloise, C. G., Timko, M. L., \& Baumgaertner, A. (1994). Working memory and inference revision in brain-damaged and normally aging adults. Journal of Speech and Hearing Research, 37, 896-912.

Twilley, L. C., Dixon, P., Taylor, D., \& Clark, K. (1994). University of Alberta norms of relative meaning frequency for 566 homographs. Memory and Cognition, 22, 111-126.

Vu, H., Kellas, G., Metcalf, K., \& Herman, R. (2000). The influence of global discourse on lexical ambiguity resolution. Memory and Cognition, 28, 236-252.

Vu, H., Kellas, G., \& Paul, S. T. (1998). Sources of sentence constraint on lexical ambiguity resolution. Memory and Cognition, 26, 979-1001.

Wapner, W., Hamby, S., \& Gardner, H. (1981). The role of the right hemisphere in the apprehension of complex linguistic materials. Brain and Language, 14, 15-33.

Wechsler, D. W. (1987). Wechsler memory scale-revised. San Antonio: Harcourt, Brace and Jovanovich.

Wiig, E. H., \& Secord, W. A. (1989). Test of language competenceexpanded edition. San Antonio: Harcourt, Brace and Jovanovich.

Wilson, B., Cockburn, J., \& Halligan, P. (1987). Behavioural inattention test. Titchfield, UK: Thames Valley Test Company.

Yorkston, K. M., \& Beukelman, D. R. (1980). An analysis of connected speech samples of aphasic and normal speakers. Journal of Speech and Hearing Disorders, 45, 27-36. 